\title{
A Molecular Dynamics Study of Small Gas Molecules Diffusion Through Composite Polysulfone/Carbon Nanotube and Polysulfone / Graphene Membranes
}

\author{
SORIN MURARU ${ }^{1}$, MARIANA IONITA ${ }^{1,2 *}$ \\ ${ }^{1}$ University Politehnica of Bucharest, Advanced Polymer Materials Group, 1-7 Gh Polizu Str., 011061, Bucharest, Romania \\ ${ }^{2}$ University Politehnica of Bucharest,Faculty of Medical Engineering, 1-7 Gh Polizu, 011061, Bucharest, Romania
}

Abstract. By using molecular dynamics, this study evaluates and compares the diffusion coefficients of $\mathrm{CH}_{4}, \mathrm{CO}_{2}, \mathrm{H}_{2}, \mathrm{~N}_{2}$ and $\mathrm{O}_{2}$ gas molecules through a polysulfone / carbon nanotube, polysulfone / graphene and polysulfone / carbon nanotube-graphene composite membranes. Mechanical properties of the composites were assessed by performing virtual traction tests and the Young's moduli exhibits values between $2.59 \mathrm{GPa}$ for polysulfone membrane and $3.87 \mathrm{GPa}$ for polysulfone / carbon nanotube-graphene composite membrane. The latter is found to exhibit superior permeability and mechanical properties, making it a promising device for gas separation.

Keywords: polysulfone, carbon nanotubes, graphene, molecular dynamics

\section{Introduction}

Gas separation field is gaining a lot of attention due to its potential applications in the energy sector, in improving the air quality and even for more daring ambitions such as space colonization. According to Brunetti, Barbieri and Drioli [1], specifically, hydrogen separation is extensively sought after in the energy sector. Likewise, this is true for methane gas and carbon dioxide, both of which also play an important role in air quality. Air separation explicitly targets the filtering of nitrogen and oxygen. Air toxicity has been found to be one of the leading factors for mortality worldwide in a recent report and this should be seen as a cause for concern as more than $90 \%$ of people worldwide live in areas exceeding the World Health Organization Guideline for healthy air [2]. Although many of the aforementioned elements can be filtered by various technologies, separation membranes are very promising devices that would heavily reduce energy costs for operation, waste and amount of machinery. Polysulfone (PSF) has long been used in the design of various commercial membrane systems due to its chemical stability, processability in different forms and moderately low mechanical properties [3]. More precisely, polysulfone on its own or as part of a mixed matrix membrane is widely used in applications related to small molecules separation processes such as hemodialysis and gas separation [4]. It is well known to be effective in the separation of small gas molecules. Likewise, graphene membranes have been extensively studied due to their mechanical strength, chemical stability and tunable permeability. They have been used in designs such as nanoporous membranes, multilayer sheets creating interlayer galleries and channels or simply as part of larger composite membranes [5]. Similarly to graphene, carbon nanotubes have been investigated as part of gas separation membranes [6]. It has been found that gas molecules would have a very fast transport rate through the carbon nanotubes. Each of the previously described materials has its own merits when it comes to small gas molecules separation, making them suitable as potential membranes. However, several limitations have to be overcome for practical applications. This study expects that by combining the processability and easy scalability associated with PSF and excellent permeability and mechanical features associated with graphene and carbon nanotubes some of the limitations related to each of the components can be surmounted.

\footnotetext{
*email: mariana.ionita@polimi.it
} 
Computational methods are used to study theoretical membrane models at a very detailed level, usually impossible to achieve through experimental methods. The most popular method to study the kinetics of small gas molecules through membranes is molecular dynamics, which gives accurate and precise insights into the properties of such a system. Composite membranes based on the aforementioned components have been investigated for various separation processes. Kim et al. [7] evaluated a composite membrane made of carbon nanotubes and polysulfone which was found to be very effective for separating various gas mixes. Likewise, membranes with graphene and polysulfone [8] have been found to have a much higher water flux than polysulfone alone [9]. This study aims to improve on the current progress on composite membranes, by incorporating both carbon nanotubes and graphene in a polysulfone membrane, expecting enhanced properties.

This article examines the gas diffusion properties of four different membrane systems, based on polysulfone, graphene and carbon nanotubes. The gases investigated are $\mathrm{H}_{2}, \mathrm{O}_{2}, \mathrm{CO}_{2}, \mathrm{CH}_{4}$ and $\mathrm{N}_{2}$. Each of these is simultaneously part of a membrane arrangement which consists of: polysulfone alone, polysulfone and carbon nanotubes (PSF-CNT), polysulfone and graphene (PSF-G), polysulfone and both carbon nanotubes and graphene (PSF-CNT-G). The diffusion of these molecules is simulated through molecular dynamics and furthermore each system's mechanical properties are calculated. The obtained results support the PSF-CNT-G membrane as promising for the efficient separation of $\mathrm{H}_{2}$ molecules.

\section{Materials and methods}

Four different systems were computationally generated and investigated, each having the same amount of small gas molecules and the same polysulfone chain consisting of 70 repeating units. In the first case, the system contains only the aforementioned molecules, while in the ensuing scenarios the setup includes additional elements in the following order: one carbon nanotube, graphene, both the carbon nanotube and graphene. The latter element consists of 147 carbon atoms passivated with 33 hydrogen atoms at the edges, while the other is a $22.54 \AA$ long carbon nanotube having a diameter of $6.21 \AA$ and made out of 168 carbon atoms passivated with 18 hydrogen atoms at the edges. These constituent elements with their respective details are shown in Figure 1.

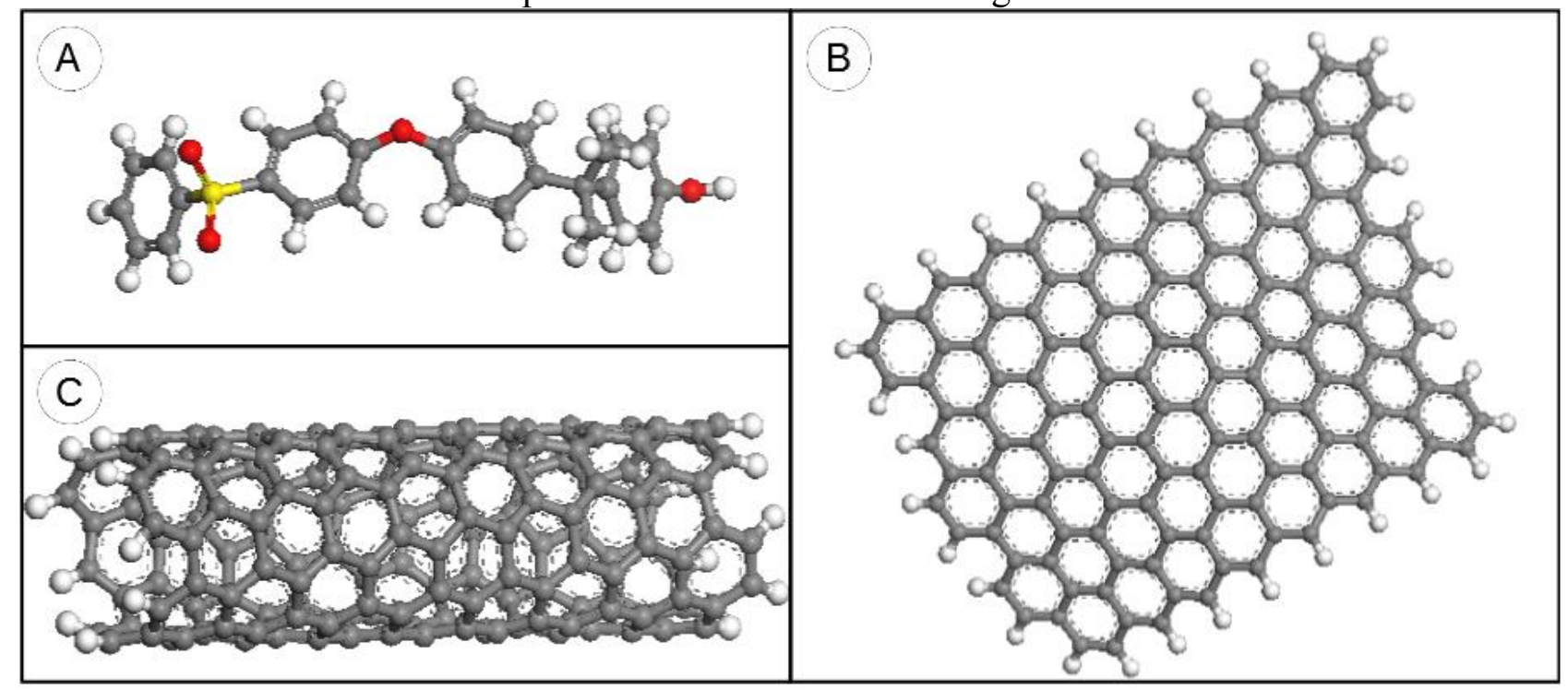

Figure 1. A) Polysulfone repeating unit (Grey atoms - carbon, White atoms - hydrogen, Red atoms oxygen, Yellow atom - Sulphur). B) Graphene sheet (Gray atoms - carbon, White atoms - hydrogen).

C) Carbon nanotube (Gray atoms - carbon, White atoms - hydrogen)

Each of the last three systems are a nanocomposite membrane with specific properties. Apart from the PSF, the carbon nanotube and the graphene sheet, there are 50 small gas molecules in each scenario, specifically ten of each: $\mathrm{CH}_{4}, \mathrm{CO}_{2}, \mathrm{H}_{2}, \mathrm{~N}_{2}$ and $\mathrm{O}_{2}$. The details of each membrane are shown in the Table 1. 
Table 1. Specific properties of each membrane type

\begin{tabular}{|ccccc|}
\hline Membrane type & $\begin{array}{c}\text { Concentration of CNT } \\
\mathbf{( \% )}\end{array}$ & $\begin{array}{c}\text { Concentration of } \\
\text { graphene (\%) }\end{array}$ & $\begin{array}{c}\text { Cubic lattice size of one } \\
\text { side (文) }\end{array}$ & $\begin{array}{c}\text { Total number of atoms } \\
\text { (atoms) }\end{array}$ \\
\hline PSF & 0 & 0 & 36.37 & 3,922 \\
\hline PSF-CNT & 4.68 & 0 & 36.17 & 4,108 \\
\hline PSF-G & 0 & 4.54 & 36.37 & 4,102 \\
\hline PSF-CNT-G & 4.48 & 4.40 & 38.88 & 4,288 \\
\hline
\end{tabular}

The molecular dynamics simulations were performed in Materials Studio, using the Discover module, in order to predict the separation of small gases through the studied membranes. The interatomic interactions are computed according to the Condensed-phase Optimized Molecular Potentials for Atomistic Simulation Studies (COMPASS) forcefield. The latter is an ab initio forcefield used for simulating accurate gas-phase and condensed-phase properties, which has been parameterized, tested and validated for the most common organic and inorganic materials [10-12]. The simulations were carried out in a cubic box with periodic boundary conditions applied in all directions. In order to relax the system, a multi step process has been applied, previously proposed by Hofmann et al. [13]. Each model in part was subjected to various subsequent energy minimization and molecular dynamics sessions in order to optimize and naturalize the state of the system. Specifically, each energy minimization session was followed by a molecular dynamics session, after which the forcefield was scaled up by a factor of 10 . The initial scale of the forcefield for the first energy minimization sessions was set at $10^{-4}$, which essentially resulted in five such sessions in total. The van der Waals interactions cutoff distance was initially set to $9.50 \AA$, and later changed to $11.50 \AA$ during the fifth energy minimization session and for all the subsequent computations. In contrast, the electrostatic interaction cutoff distance was held constant at $9.50 \AA$. The temperature control method in the molecular dynamics sessions used was the Andersen method, in an NVT ensemble at $298 \mathrm{~K}$ for $0.2 \mathrm{~ns}$, with a timestep of 1 fs. The PSF-CNT-G membrane system with randomly scattered small gas molecules after the equilibration process is shown in the Figure 2.

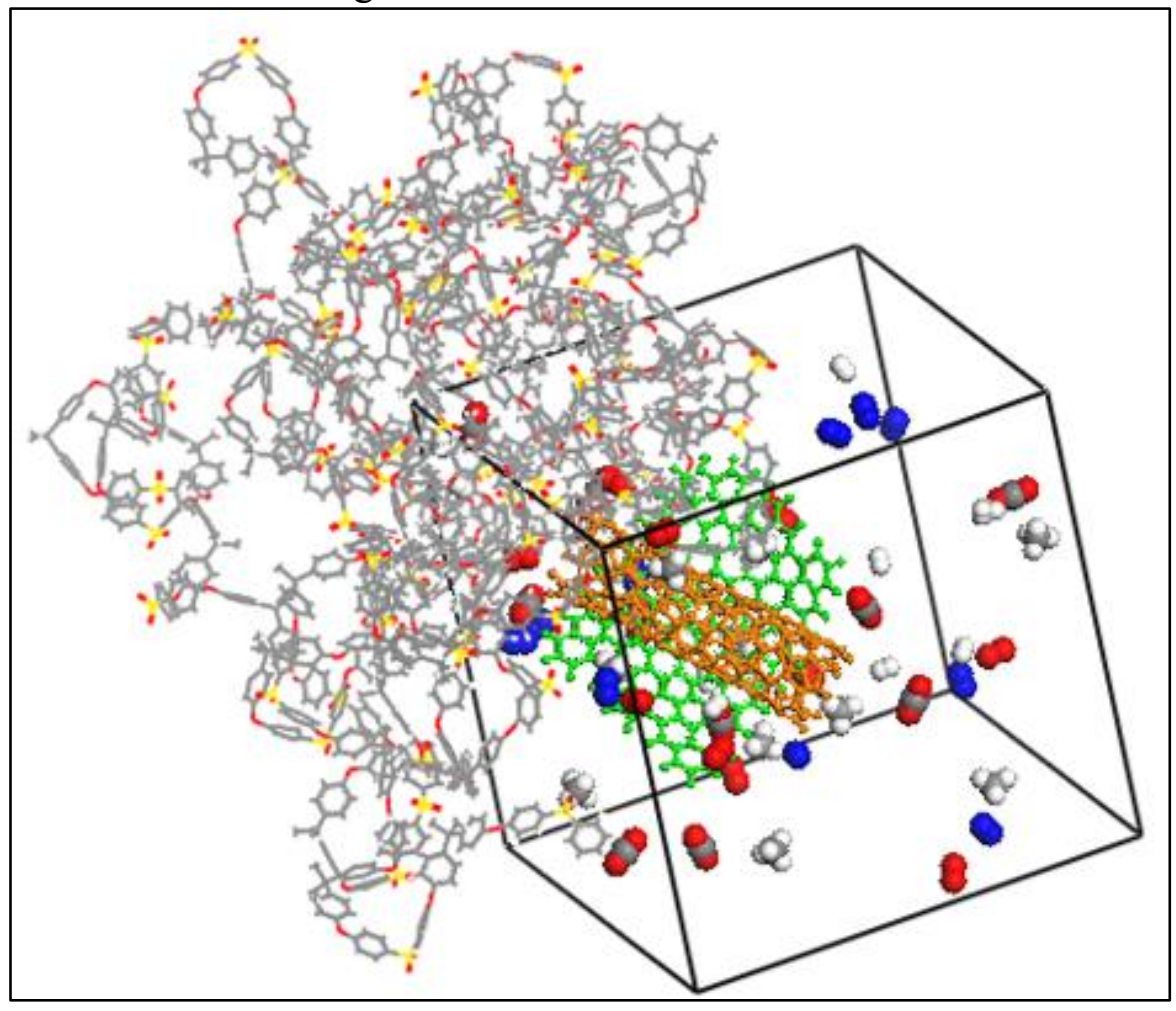

Figure 2. The PSF-CNT-G membrane system with randomly scattered small gas molecules. The carbon nanotube is displayed in orange, while the graphene sheet is displayed in green. The gas molecules are displayed as $\mathrm{CPK}$ and appear larger for better visualization 
The molecular dynamics simulations, launched after the energy optimization, were performed for $800 \mathrm{~ns}$ in an NVE ensemble at an initial temperature of $298 \mathrm{~K}$ with $1 \mathrm{fs}$ timestep and frames recorded every 5 ps. The van der Waals cutoff value was set at $9.50 \AA$ and the electrostatic interactions were cutoff at $11.00 \AA$. The diffusion coefficients were found applying Einstein's diffusion equation:

$$
D=\lim _{t \rightarrow \infty} \frac{\left|\overline{\vec{r}(t)-\vec{r}^{+}(0)}\right|^{2}}{2 d_{i} t}
$$

where $\mathrm{D}$ is the diffusion coefficient, $\mathrm{r}$ is the position vector, $\mathrm{d}_{\mathrm{i}}$ is the number of spatial dimensions and $\mathrm{t}$ is the simulation time. The line above the denominator means the average of the entity, which is obtained by finding the gradient of the linear mean square displacement using the Forcite module in Materials Studio. Furthermore, the Forcite module was used to extract the mechanical properties of each of the four systems.

\section{Results and discussions}

Polysulfone is a promising membrane, given its chemical and thermal stability [14]. Equally, graphene has been widely investigated for gas separation given its superior mechanical strength and high chemical robustness [15]. Moreover, carbon nanotubes are known in particular for their rapid mass transport [16]. Figure 3 shows the initially generated membrane containing only PSF at the starting point of the energy minimization process and the optimized membrane containing only PSF at the end of the energy minimization process.

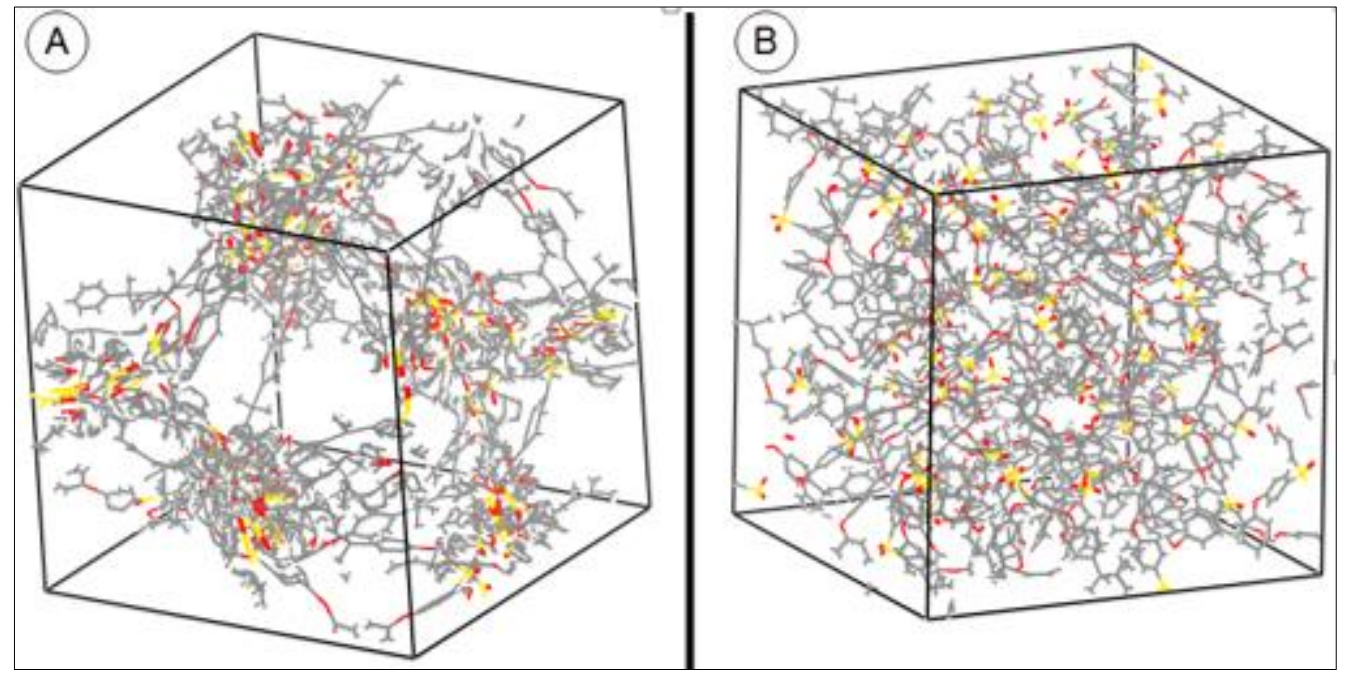

Figure 3. A) The initial PSF membrane. B) The optimized PSF membrane

The initial PSF membrane is not uniformly distributed in the cubic lattice and there are many significantly large pores or simply free volume. In contrast, the optimized PSF membrane presents well distributed PSF chains in the whole lattice, achieving a roughly isotropic material. Figure 4 shows the total energy evolution in the last energy minimization process and additionally, the total energy evolution in the subsequent molecular dynamics session for the PSF-CNT-G membrane, indicating the successful equilibration for the computational model. Similar energy profile were obtained for the other computational systems. 

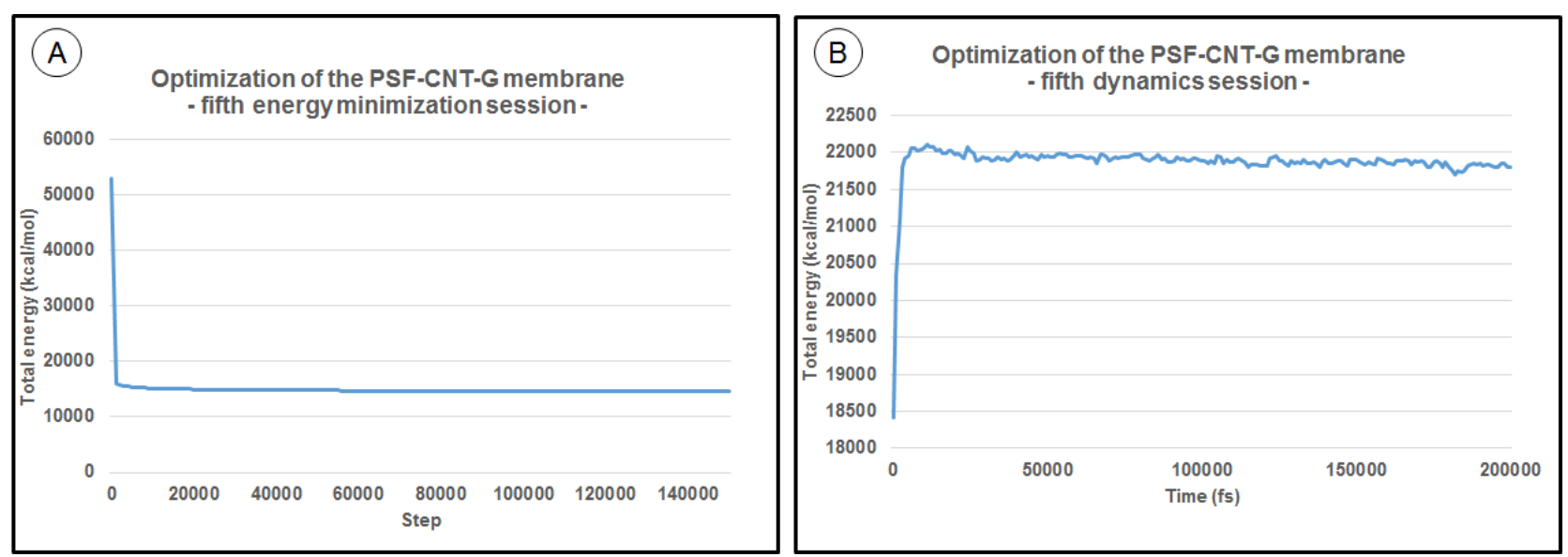

Figure 4. A) Total energy ( $\mathrm{kcal} / \mathrm{mol})$ evolution in the fifth energy minimization session for the PSF-CNT-G membrane. B) Total energy ( $\mathrm{kcal} / \mathrm{mol})$ evolution in the fifth molecular dynamics session for the PSF-CNT-G membrane

Using the Forcite module, the mechanical properties of the membranes has been computed. Given that the PSF membrane is approximately isotropic, its overall Young's modulus has been calculated to be $2.59 \mathrm{GPa}$. This is seemingly supported by the findings of Linares and Acosta [17] who measured this to be around $1.53 \mathrm{GPa}$. However, given the fact that they tested an experimental model and in this study a theoretical model is employed, the lower value is expected given possible defects or irregularities of the real material. Therefore, the $2.59 \mathrm{GPa}$ value is considered valid. Table 2 presents the Young's modulus found for each of the studied membranes.

Table 2. The Young's modulus for each type of membrane

\begin{tabular}{|c|c|c|c|c|c|}
\hline Membrane type & $\begin{array}{c}\text { Considered spatial } \\
\text { direction }\end{array}$ & $\begin{array}{l}\text { Young's modulus } \\
\text { (GPa) }\end{array}$ & $\begin{array}{c}\text { Concentration of } \\
\text { CNT }(\%)\end{array}$ & $\begin{array}{l}\text { Concentration of } \\
\text { graphene }(\%)\end{array}$ & $\begin{array}{c}\text { Cubic lattice size } \\
\text { of one side }(\AA)\end{array}$ \\
\hline PSF & Isotrope & 2.59 & 0 & 0 & 36.37 \\
\hline \multirow{3}{*}{ PSF-CNT } & $\mathrm{X}$ & 3.41 & \multirow{3}{*}{4.68} & \multirow{3}{*}{0} & \multirow{3}{*}{36.17} \\
\hline & $\mathrm{Y}$ & 2.85 & & & \\
\hline & $\mathrm{Z}$ & 2.92 & & & \\
\hline \multirow{3}{*}{ PSF-G } & X & 1.94 & \multirow{3}{*}{0} & \multirow{3}{*}{4.54} & \multirow{3}{*}{36.37} \\
\hline & $\mathrm{Y}$ & 4.35 & & & \\
\hline & Z & 2.38 & & & \\
\hline \multirow{3}{*}{ PSF-CNT-G } & $\mathrm{X}$ & 2.97 & \multirow{3}{*}{4.48} & \multirow{3}{*}{4.40} & \multirow{3}{*}{38.88} \\
\hline & $\mathrm{Y}$ & 2.44 & & & \\
\hline & Z & 3.87 & & & \\
\hline
\end{tabular}

The Young's modulus found for each composite membrane is considered within the expected limits as it modifies the directional value according to the placement of the reinforcing agents. For the PSFCNT membrane the overall increase is about $18 \%$. Maphuta et al. [18] found a $77 \%$ increase in the Young's modulus in a similar composite membrane with $7.50 \%$ concentration of carbon nanotubes. The difference may come from the slightly lower concentration in this study's membrane and the explicitly much higher initial modulus found in the PSF membrane. For the PSF-G membrane the overall increase is approximately $11 \%$, while for the last membrane the incremental difference is about $19 \%$. Figure 5 shows the optimized PSF-CNT, PSF-G and PSF-CNT-G membranes. 

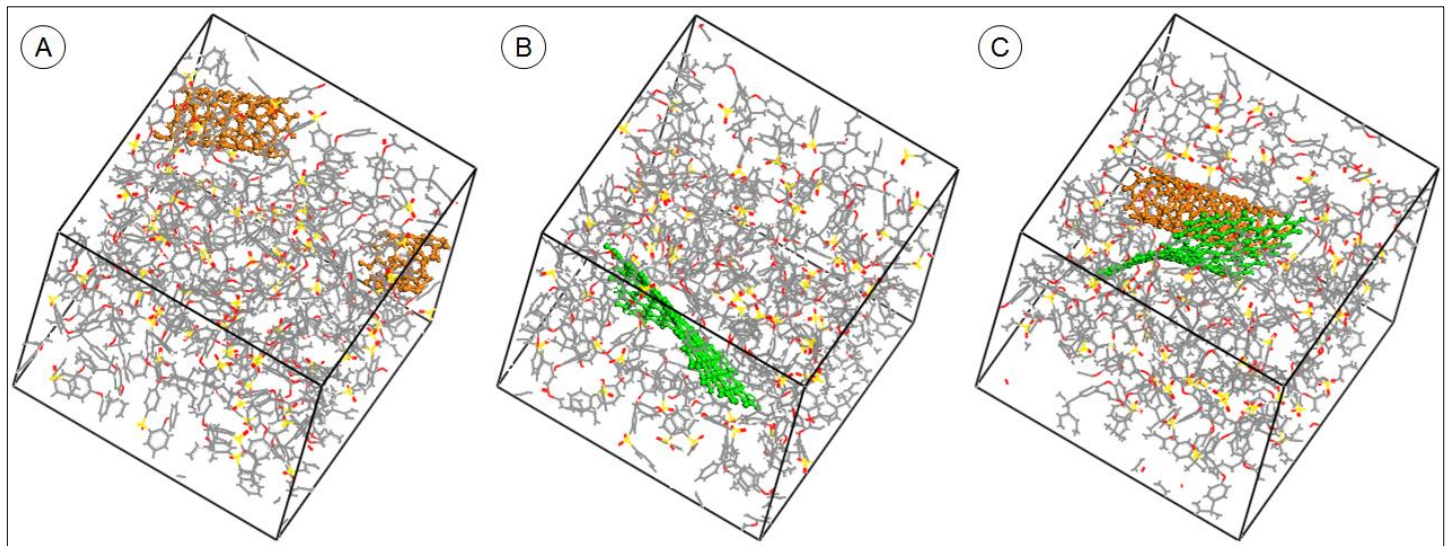

Figure 5. A) Optimized PSF-CNT membrane. B) Optimized PSF-G membrane. C) Optimized PSF-CNT-G membrane

The mean square displacements were found by analyzing the trajectories of each of the small gas molecules obtained in the molecular dynamics simulations. Specifically, this was done by using the Forcite module in Materials Studio. Afterwards, the Einstein equation for diffusion was used to obtain the diffusion coefficients for each of the small gas molecules, which are presented in Table 3.

Table 3. Diffusion coefficients of each gas molecule for each membrane

\begin{tabular}{|c|ccc|}
\hline Gas molecule & PSF & Diffusion coefficient $\left(\mathrm{cm}^{2} / \mathbf{s}\right)$ in membrane \\
& PSF-CNT & PSF-G & PSF-CNT-G \\
\hline $\mathrm{CH}_{4}$ & $(0.53 \pm 0.18) \times 10^{2}$ & $(0.70 \pm 0.24) \times 10^{2}$ & $(1.40 \pm 0.70) \times 10^{2}(1.97 \pm 0.77) \times 10^{3}$ \\
\hline $\mathrm{CO}_{2}$ & $(0.10 \pm 0.05) \times 10^{2}$ & $(1.33 \pm 0.70) \times 10^{2}$ & $(0.93 \pm 0.42) \times 10^{2}(1.35 \pm 0.37) \times 10^{3}$ \\
\hline $\mathrm{H}_{2}$ & $(2.42 \pm 0.70) \times 10^{4}$ & $(2.87 \pm 1.24) \times 10^{4}$ & $(1.29 \pm 0.36) \times 10^{4}(5.46 \pm 1.83) \times 10^{4}$ \\
\hline $\mathrm{N}_{2}$ & $(0.85 \pm 0.39) \times 10^{2}$ & $(0.87 \pm 0.27) \times 10^{2}$ & $(4.11 \pm 1.46) \times 10^{2}(0.83 \pm 0.37) \times 10^{3}$ \\
\hline $\mathrm{O}_{2}$ & $(2.06 \pm 1.18) \times 10^{2}$ & $(2.83 \pm 0.87) \times 10^{2}$ & $(2.44 \pm 1.75) \times 10^{2}(3.00 \pm 1.41) \times 10^{3}$ \\
\hline
\end{tabular}

Hydrogen molecules have the highest diffusion coefficient explained by their smallest molecular diameter. All of the other gas molecules have at least one order of magnitude smaller diffusion coefficients. There is no stable hierarchy that applies to all of the membranes, however a rough pattern is emerging concerning having the oxygen as the follow-up runner. Although it would be expected for the diffusion coefficients to decrease given the addition of obstacles in the PSF membrane, such as the carbon nanotube or graphene sheet, this rule applies to only for some of the gas molecules and does not hold true for all of them. Moreover, in the PSF-CNT-G membrane, all diffusion coefficients have increased in comparison to any other membrane, being the largest out of all the studied scenarios. The intuition behind this increase might be linked to the fact that the graphene sheet and the carbon nanotube seem to agglomerate towards the same area, effectively creating a free volume and therefore significantly increase the permeability of the membrane overall. This means that the addition of carbon nanotubes and graphene sheets improves the mechanical properties of the system, while enhancing the diffusion through the membrane.

\section{Conclusions}

This study has evaluated the gas permeability of the PSF-CNT-G membrane in comparison to three other membranes, namely PSF, PSF-CNT and PSF-G. It has been found that by adding both carbon nanotubes and graphene into the polysulfone membrane, the diffusion coefficients of the studied gases $\mathrm{CH}_{4}, \mathrm{CO}_{2}, \mathrm{H}_{2}, \mathrm{~N}_{2}$ and $\mathrm{O}_{2}$ have all increased significantly. This could be explained by the free volume 
created from the agglomeration tendency of the graphene sheet and the carbon nanotube, effectively reducing the barriers for the gas molecules. As expected, hydrogen is found to be the molecule with the highest diffusion rate at $4.45 \times 10^{4} \mathrm{~cm}^{2} / \mathrm{s}$, given its smallest molecular diameter. The addition of the carbon nanotube and the graphene, which notably act as reinforcing agents, improves the mechanical properties of the membrane. Overall, the composite membrane PSF-CNT-G is found to have superior properties for gas diffusion and mechanical attributes in comparison to either PSF, PSF-CNT or PSF-G membranes.

Acknowledgment:This work was supported by a grant of the Executive Agency for Higher Education, Research, Development and innovation funding (UEFISCDI), project number PN-III-P1-1.1-TE-201624-2, contract TE 122/2018.

\section{References}

1. BRUNETTI, A., BARBIERI, G., DRIOLI, E., Encyclopedia of Membrane Science and Technology, John Wiley \& Sons, New Jersey, 2013

2. HEALTH EFFECTS INSTITUTE, State of Global Air 2019: A Special Report on Global Exposure to Air Pollution and its Disease Burden, Health Effects Institute, Boston, 2019.

3. HUANG, H., YANG, S., J. Aerosol Sci., 37, nr. 10, 2006, p. 1198.

4. JULIAN, H., WENTEN, I.G., IOSR J. Eng., 2, nr. 3, 2012, p. 484.

5. YOON, H., CHO, Y., PARK, H., Philos. T. Roy. Soc. A, 374, nr. 2060, 2016, p. 20150024.

6. SEARS, K., DUMÉE, L., SCHÜTZ, J., SHE, M., HUYNH, C., HAWKINS, S., DUKE, M., GRAY, S., Materials, 3, nr. 1, 2010, p. 127.

7. KIM, S., JINSCHEK, J.R., CHEN, H., SHOLL, D.S., MARAND, E., Nano. Lett., 7, nr. 9, 2007, p. 2806.

8. KAUSAR, A., Mater. Res. Innov., 2018, p.1.

9. YE, Y.S., TSENG, C.Y., SHEN, W.C., WANG, J.S., CHEN, K.J., CHENG, M.Y., RICK, J., HUANG, Y.J., CHANG, F.C., HWANG, B.J., J. Mater. Chem., 21, 2011, p. 10448.

10. SHAN, M., XUE, Q., JING, N., LING, C., ZHANG, T., YAN, Z., ZHENG, J., Nanoscale, 4, nr. 17, 2012, p. 5477.

11. SUN, H., J. Phys. Chem. B, 102, nr. 38, 1998, p. 7338.

12. RIGBY, D., SUN, H., EICHINGER, B., Polym. Int., 44, nr. 3, 1997, p. 311.

13. HOFMANN, D., FRITZ, L., ULBRICH, J., SCHEPERS, C., BÖHNING, M., Macromol. Theor. Simul., 9, 2000, p. 293-327.

14. MICULESCU, M., THANKUR, V.K., MICULESCU, F., VOICU, S., Polym. Advan. Technol., 27, nr. 7, 2016, p. 844.

15. LEE, C., WEI, X., KYSAR, J.W., HONE, J., Science, 321, 2008, p. 385.

16. SANIP, S.M., ISMAIL, A.F., GOH, P.S., SOGA, T., TANEMURA, M., YASUHIKO, H., Sep. Purif. Technol., 78, nr. 2, 2011, p. 208.

17. LINARES, A., ACOSTA, J., J. Appl. Polym. Sci., 92, nr. 5, 2004, p. 3030.

18. MAPHUTA, S., MOOTHI, K., MEYYAPPAN, M., IYUKE, S., Sci. Rep., 3, nr. 1, 2013

$\overline{\text { Manuscript received: } 26.11 .2019}$ 